\title{
Evaluation of a patient-specific cost function to predict the influence of foot path on the knee adduction torque during gait
}

\author{
BENJAMIN J. FREGLY $\dagger+\uparrow *$, JEFFERY A. REINBOLT $\dagger$ and TERESE L. CHMIELEWSKI§ \\ $†$ Department of Mechanical \& Aerospace Engineering, University of Florida, Gainesville, FL, USA \\ $\ddagger$ Department of Biomedical Engineering, University of Florida, Gainesville, FL, USA \\ ๆ Department of Orthopaedics and Rehabilitation, University of Florida, Gainesville, FL, USA \\ $\S$ Department of Physical Therapy, University of Florida, Gainesville, FL, USA
}

\begin{abstract}
A large external knee adduction torque during gait has been correlated with the progression of knee osteoarthritis (OA). Though foot path changes (e.g. toeing out) can reduce the adduction torque, no method currently exists to predict whether an optimal foot path exists for a specific patient. This study evaluates a patient-specific optimization cost function to predict how foot path changes influence both adduction torque peaks. Video motion and ground reaction data were collected from a patient with knee OA performing normal, toe out, and wide stance gait. Joint and inertial parameters in a dynamic, 27 degree-of-freedom, full-body gait model were calibrated to the patient's normal gait data. The model was then used in gait optimizations that predicted how the patient's adduction torque peaks would change due to changes in foot path. The cost function tracked the patient's normal gait data using weight factors calibrated to toe out gait and tested using wide stance gait. For both gait motions, the same cost function weights predicted the change in both adduction torque peaks to within $7 \%$ error. With further development, this approach may eventually permit the design of patient-specific rehabilitation procedures such as an optimal foot path for patients with knee OA.
\end{abstract}

Keywords: Patient-specific cost function; Optimization; Gait; Foot path; Knee adduction moment

\section{Introduction}

As computational technologies continue to improve, dynamic musculoskeletal models may eventually permit optimization of functional outcome for surgical or rehabilitative interventions on an individual patient basis. An important step toward this goal is the development of musculoskeletal models that match key features of the patient's anatomy and movement characteristics. These features include: (1) the kinematic structure of the patient, as represented by the types, positions, and orientations of functional axes in the body segments (Sommer and Miller 1980, Bogert et al. 1994, Reinbolt et al. 2005), (2) the inertial properties of the patient, as indicated by segment masses, mass centres, and moments of inertia (Vaughan et al. 1982, de Leva 1996, Rao et al. 2006), and (3) the control strategy used by the patient (Buchanan et al. 2004, Liu et al. 2005, Bottasso et al. 2006). If all three features could be calibrated to pretreatment movement data collected from a patient, the calibrated model could be used to optimize functional outcome for the various treatment scenarios and parameters under consideration.

The control strategy feature is the most difficult one to calibrate to a patient's movement data, since a wide variety of approaches could be used to model a patient's unique control characteristics. One approach is to develop a patient-specific neural control model based on excitation signals sent to individual muscle actuators. Recent studies using optimization methods and EMG-based controllers have made significant progress in this area (Anderson and Pandy 2001, Thelen et al. 2003, Buchanan et al. 2004). Apart from the assumed controller structure, one of the difficulties with this approach is developing methods to determine patient-specific muscle-tendon model parameters (e.g. peak isometric force, optimal muscle fiber length, tendon slack length; Zajac 1989, Garner and Pandy 2003, Lloyd and Besier 2003) and moment arms (Arnold et al. 2000, Murray et al. 2002) for each muscle included in the larger musculoskeletal model. A simpler approach is to develop control laws based on net joint torques calculated from inverse dynamics and on other external

*Corresponding author. Email: fregly@ufl.edu 
data that can be measured from the specific patient (Suzuki et al. 1996, Wada et al. 2001, Liu et al. 2005). Though less powerful than methods based on control of individual muscles, such an approach could allow clinicians to predict how a proposed treatment would alter a patient's joint motions and loads for some subset of clinically significant conditions.

This study investigates whether a patient-specific optimization cost function based on external gait measurements can be used to predict how changes in foot path affect the external knee adduction torque during gait. Since the magnitude of this torque has been positively correlated with medial compartment knee load (Zhao et al. 2007) and the progression of medial compartment knee osteoarthritis (OA; Baliunas et al. 2002, Miyazaki et al. 2002), rehabilitation treatments that lower this torque may be useful for slowing the progression of knee OA. The patient-specific cost function is defined by adjustable weight factors on error terms that track a patient's normal kinematic and kinetic gait data. The goal is to find weights such that minimization of the cost function produces the patient's knee adduction torque curve for gait motions with altered foot paths. The work presented here is the first step toward the development of a computational approach for designing patient-specific rehabilitation procedures, such as an optimal foot path for patients with knee OA.

\section{Methods}

\subsection{Experimental gait data}

Experimental gait data were collected from a patient with knee OA (male, age 40 years, height $170 \mathrm{~cm}$, mass $69 \mathrm{~kg}$ ) using a video-based motion analysis system with modified Cleveland Clinic marker set (Motion Analysis Corporation, Santa Rosa, CA) and two force plates (AMTI, Watertown, MA). The modification involved adding three markers to each foot segment to allow three-dimensional tracking of the feet. Institutional review board approval and informed consent were obtained prior to the experiments. For all trials, the patient walked at a selfselected speed of $1.4 \mathrm{~m} / \mathrm{s}$. Unloaded isolated joint motions were performed to exercise the primary functional axes of each lower extremity joint (hip, knee and ankle on each side). For each joint, the patient was instructed to move the distal segment within the physiological range of motion so as to exercise all degrees-of-freedom (DOFs) of the joint (Reinbolt et al. 2005). Gait motion and ground reaction data were collected to provide simultaneous motion of all lower extremity joints under load-bearing physiological conditions. One cycle (i.e. left heel strike to left heel strike) of three different gait motions (normal, toe out, and wide stance) was analysed to produce a range of different knee adduction torque curves for testing the predictive capabilities of the proposed patient-specific cost function.

\subsection{Patient-specific gait model}

A dynamic, patient-specific gait model was developed to predict new gait motions and loads given experimental data for the patient's normal gait motion. The full-body model is three-dimensional and possesses 27 DOFs (figure 1). The equations of motion for the model were derived using two different methods-the symbolic manipulation software Autolev ${ }^{\mathrm{TM}}$ (OnLine Dynamics, Sunnyvale, CA) and the musculoskeletal modeling software SIMM with the Dynamics Pipeline (Motion Analysis Corporation, Santa Rosa, CA), thereby allowing us to verify the accuracy of the equations. Comparable to Anderson and Pandy's (2001) model structure, three translational and three rotational DOFs express the movement of the pelvis in the laboratory reference frame, and the remaining 13 segments comprise four open chains branching from the pelvis. The kinematic structure of the model is defined by joint parameters that designate the positions and orientations of joint axes within adjacent segment coordinate systems. The kinematic structure utilizes the following joint types: three DOF hip, one DOF knee (with external reaction torque calculated for adduction), two DOF ankle (nonintersecting axes; Bogert et al. 1994), three DOF back, two DOF shoulder, and one DOF elbow. The inertial properties of the model are defined by inertial parameters that designate each

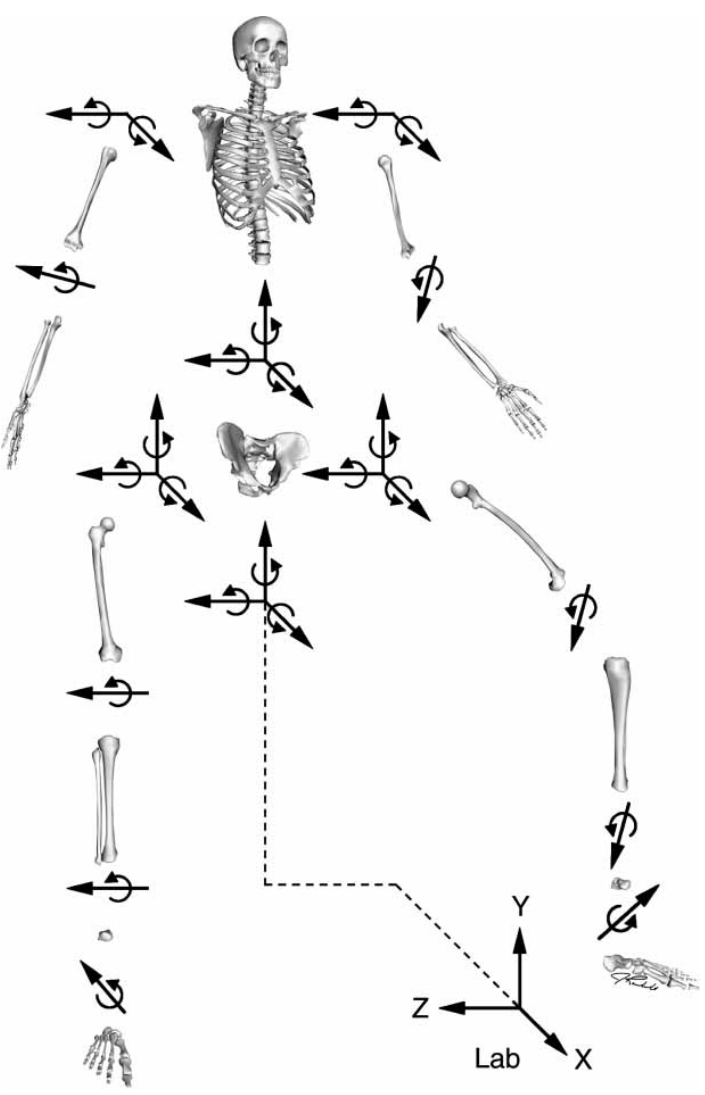

Figure 1. Schematic of the three-dimensional, 14 segment, 27 degreeof-freedom full-body gait model with segments connected by gimbal, universal, and pin joints. 
segment's mass, mass centre along its longitudinal axis, and three central principal moments of inertia.

All joint and inertial parameters in the model were calibrated to the patient's normal gait data and isolated joint motion experiments. First, values of the joint parameters defining the hip, knee and ankle joint in each leg were identified using optimization of the kinematic data from the unloaded isolated joint motion trials (Reinbolt et al. 2005). Next, these values were adjusted slightly via optimization to match the kinematic data from the loaded normal gait trial. We used this two-stage approach since gait trials do not exercise the full range of motion for each lower extremity joint. Finally, values of the inertial parameters for all segments in the model were identified using optimization of the kinetic data from the normal gait trial (Fregly et al. 2007). Matlab's nonlinear least squares algorithm (The Mathworks, Natick, MA) was used for all optimizations. The resulting joint and inertial parameter values were assumed to be valid for all three gait motions (normal, toe out, and wide stance) performed by the patient.

For the subsequent predictive gait optimizations, inverse rather than forward dynamics simulations were performed with the calibrated model (Mazza and Cappozzo 2004), thereby eliminating stability problems due to the use of open-loop joint torque controls. Inputs to the inverse dynamics model were values of the 27 generalized coordinates, their first and second time derivatives, and bilateral ground reaction forces and torques, while outputs were 27 joint torques (including six residual forces and torques acting on the pelvis), bilateral foot paths (not generalized coordinates), trunk orientation (also not generalized coordinates), and bilateral centres of pressure. Ground reactions were set to zero during periods when the foot was known to be in contact with the floor. The pelvis residual loads, which represent errors in the model's structure, parameters values, and/or experimental inputs, were driven close to zero through calibration of the model's joint and inertial parameters.

\subsection{Patient-specific cost function}

To predict changes in the patient's knee adduction torque due to changes in foot path, we constructed an optimization cost function containing weight factors that were calibrated to the patient's toe out gait data and then tested using his wide stance gait data. The proposed cost function assumes that different gait motions produced by the same patient will be neighbouring solutions of one another. For example, we do not expect the joint motions and torques from a patient's toe out gait motion to be markedly different from those of his normal gait motion. Thus, we assume that a patient's normal gait data provides a good initial guess for how he will walk under other conditions. This assumption leads to the formulation of an optimal tracking problem, where the cost function minimizes a weighted sum of squares of changes in kinematic and kinetic quantities away from the patient's normal gait data. If the correct weights are placed on the various quantities in the cost function, then ideally the optimization will predict how the patient would walk under conditions for which experimental data are not available.

The cost function weights were calibrated to the patient's toe out gait data by solving a two-level optimization problem. The outer-level optimization varied the weights used by the inner-level cost function so as to minimize errors between experimental and predicted adduction torque peaks for toe out gait. The outer-level cost function was defined as

$$
e=\min _{w}\left[\left(T_{1}-T_{1}^{\prime}(w)\right)^{2}+\left(T_{2}-T_{2}^{\prime}(w)\right)^{2}\right]
$$

where $e$ is the cost function value, $w$ are the six inner-level cost function weights treated as outer-level design variables, $T_{1}$ and $T_{2}$ are the first and second adduction torque peaks measured experimentally, and $T_{1}^{\prime}(w)$ and $T_{2}^{\prime}(w)$ are the first and second adduction torque peaks predicted by the inner-level optimization. Given the current guess for $w$ from the outer-level, the inner-level optimization varied the shape of the input motion and ground reaction curves so as to minimize the weighted sum of squares of errors between experimental (normal gait with adjusted foot path) and predicted (toe out gait) kinematic and kinetic quantities. The specific form of the inner-level cost function was

$$
\begin{aligned}
\left(T_{1}^{\prime}(w), T_{2}^{\prime}(w)\right)= & \min _{p}\left\{10 \sum_{i=1}^{n f} \sum_{j=1}^{6} \sum_{k=1}^{2}\left(\Delta q_{\mathrm{Foot}}^{2}(p)\right)_{i j k}\right. \\
& +w_{1} \sum_{i=1}^{n f} \sum_{j=1}^{3}\left(\Delta q_{\text {Trunk }}^{2}(p)\right)_{i j} \\
& +w_{2} \sum_{i=1}^{n f}\left[\sum_{j=1}^{6}\left(\Delta q_{\text {Pelvis }}^{2}(p)\right)_{i j}\right. \\
& \left.+\sum_{j=1}^{6} \sum_{k=1}^{2}\left(\Delta q_{\mathrm{Leg}}^{2}(p)\right)_{i j k}\right] \\
& +w_{3} \sum_{i=1}^{n f} \sum_{j=1}^{6} \sum_{k=1}^{2}\left(\Delta T_{\mathrm{Leg}}^{2}(p)\right)_{i j k} \\
& +w_{4} \sum_{i=1}^{n f} \sum_{j=1}^{3} \sum_{k=1}^{2}\left(\Delta F_{\mathrm{Ground}}^{2}(p)\right)_{i j k} \\
& +w_{5} \sum_{i=1}^{n f} \sum_{j=1}^{2} \sum_{k=1}^{2}\left(\Delta \mathrm{CoP}_{\mathrm{Foot}}^{2}(p)\right)_{i j k} \\
& \left.+w_{6} \sum_{i=1}^{n f} \sum_{j=1}^{3}\left(\Delta F_{\mathrm{Pelvis}}^{2}(p)+\Delta T_{\mathrm{Pelvis}}^{2}(p)\right)_{i j}\right\}
\end{aligned}
$$

where $p$ are 660 polynomial-Fourier coefficients treated as inner-level design variables, $w_{1}$ through $w_{6}$ are the weight factors provided by the outer-level optimizer, $\Delta q_{\text {Foot }}$ are foot path tracking errors, $\Delta q_{\text {Trunk }}$ are trunk orientation 
tracking errors, $\Delta q_{\text {Pelvis }}$ are pelvis pose tracking errors, $\Delta q_{\text {Leg }}$ are joint angle tracking errors for the legs, $\Delta T_{\text {Leg }}$ are joint torque tracking errors for the legs, $\Delta F_{\text {Ground }}$ are ground reaction force tracking errors, $\Delta \mathrm{CoP}_{\mathrm{Foot}}$ are foot centre of pressure tracking errors, and $\Delta F_{\text {Pelvis }}$ and $\Delta T_{\text {Pelvis }}$ are tracking errors for the residual forces and torques acting on the pelvis. Units are $\mathrm{mm}$ for translations, deg for rotations, $\mathrm{N}$ for force, and $\mathrm{Nm}$ for torque. A cubic polynomial plus eight Fourier harmonics (i.e. 20 coefficients per curve) was used to parameterize each motion and ground reaction curve (Nagurka and Yen 1990). The only exceptions were the motion curves for the shoulders and elbows, which were prescribed to match the patient's nominal gait data. The initial guess and tracked values for the inner-level optimization were taken as the patient's normal gait data. For tracking purposes, three fixed offsets (internal-external rotation, medial-lateral translation and anterior-posterior translation) were calculated to convert the patient's normal foot path into a toe out foot path that best matched his toe out gait data. The outer-level optimization utilized a univariate search algorithm developed by the lead author (Fregly et al. 2005) while the inner-level optimization utilized Matlab's nonlinear least squares algorithm.

Once the inner-level cost function weights were calibrated to the patient's toe out gait data, they were tested using his wide stance gait data. Testing required only the inner-level optimization, with three new foot path offsets being calculated to convert the patient's normal foot path into a wide stance foot path that best matched his wide stance gait data. Thus, while calibration required repeated inner-level optimizations to determine the innerlevel cost function weights, testing required only a single inner-level optimization to evaluate how well the same cost function weights could predict both adduction torque peaks for a different gait motion.

\section{Results}

After the cost function weights were calibrated to the patient's toe out gait data, the inner-level optimization produced close predictions of both knee adduction torque peaks. The first adduction torque peak was matched to within $7 \%$ error and the second peak to within $3 \%$ (figure 2(a)). The primary kinematic change observed experimentally, which was an increase in hip external rotation, was also well predicted (figure 3(a)). However, not all kinetic changes observed experimentally were reproduced by the optimization (figure 4(a)). While the decrease in hip abduction torque half way through the gait cycle was well predicted, the decrease in hip extension torque near the start of the cycle and the increase in knee extension torque over much of stance phase were not. The optimization predicted little change in ground reaction forces, consistent with experimental observations (figure 5(a)). It also predicted little change in the anterior-posterior centre of pressure trajectory and a gradual lateral shift in the medial-lateral centre of pressure trajectory, both consistent with experimental findings (figure 6(a)).

When the same cost function weights were tested using the patient's wide stance gait data, the inner-level optimization again produced close predictions of both knee adduction torque peaks. The first abduction torque peak was predicted to within $7 \%$ error and the second peak to within $6 \%$ (figure 2(b)). Unlike for toe out gait, the primary kinematic changes observed experimentally were not well predicted, these being an increase in hip and knee flexion over the first half of the cycle (figure 3(b)). While the hip extension torque was well predicted, the optimization again did not predict the increase in knee extension torque during stance phase, while it also predicted a spurious decrease in hip abduction torque during early stance phase (figure 4(b)). Little change in the ground reaction forces was again predicted, though the predicted increase in medial force was less than that observed experimentally (figure 5(b)). Finally, the optimization predicted little change in the anteriorposterior centre of pressure trajectory and a fixed lateral offset in the medial-lateral centre of pressure trajectory, both highly consistent with experimental findings (figure 6(b)).

\section{Discussion}

This study evaluated whether a patient-specific optimization cost function in conjunction with a patient-specific (a)

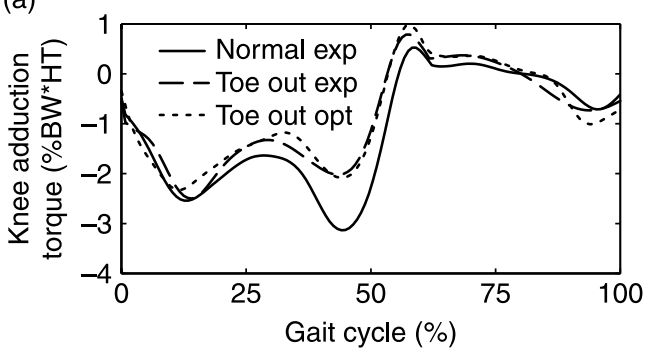

(b)

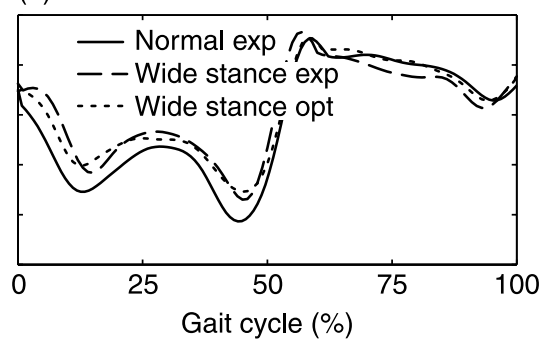

Figure 2. Evaluation of left knee adduction torque curves predicted using the patient-specific optimization cost function. Column (a) Toe out gait prediction for which the cost function weights were calibrated. Column (b) Wide stance gait prediction for which the cost function weights were tested. Solid curves are from the patient's normal experimental gait motion, dashed curves are from his toe out or wide stance experimental gait motion, and dotted curves are from the toe out or wide stance optimization predictions. 
(a)
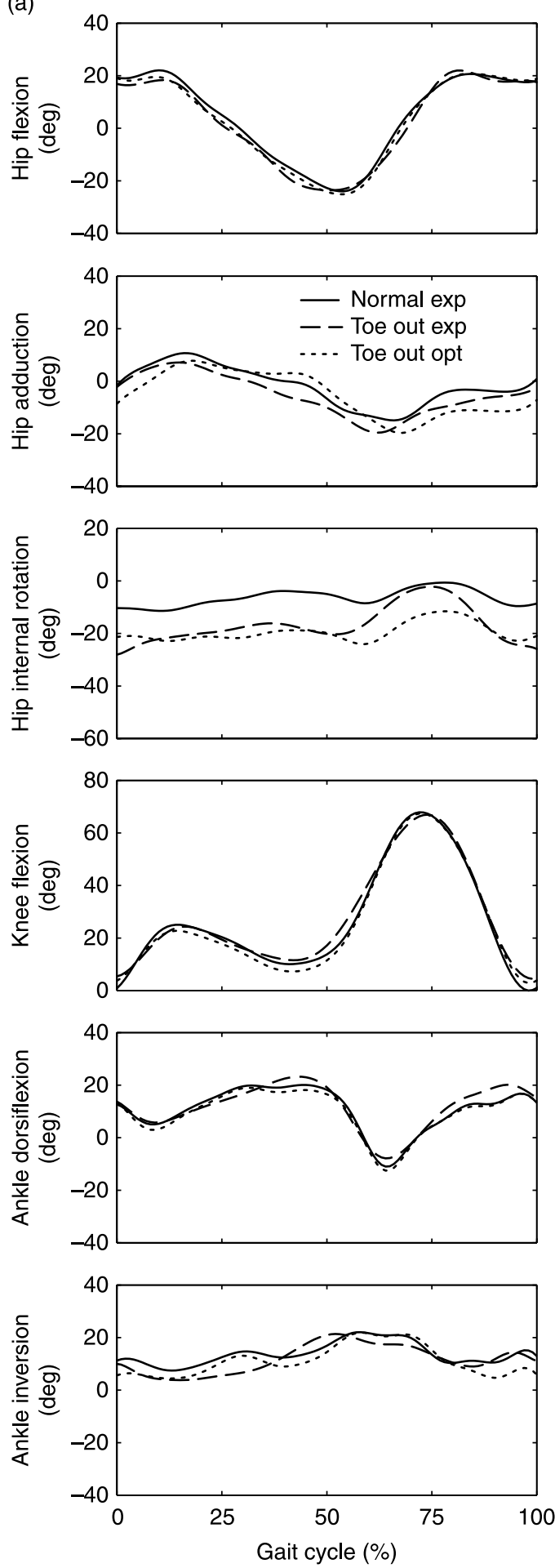

(b)
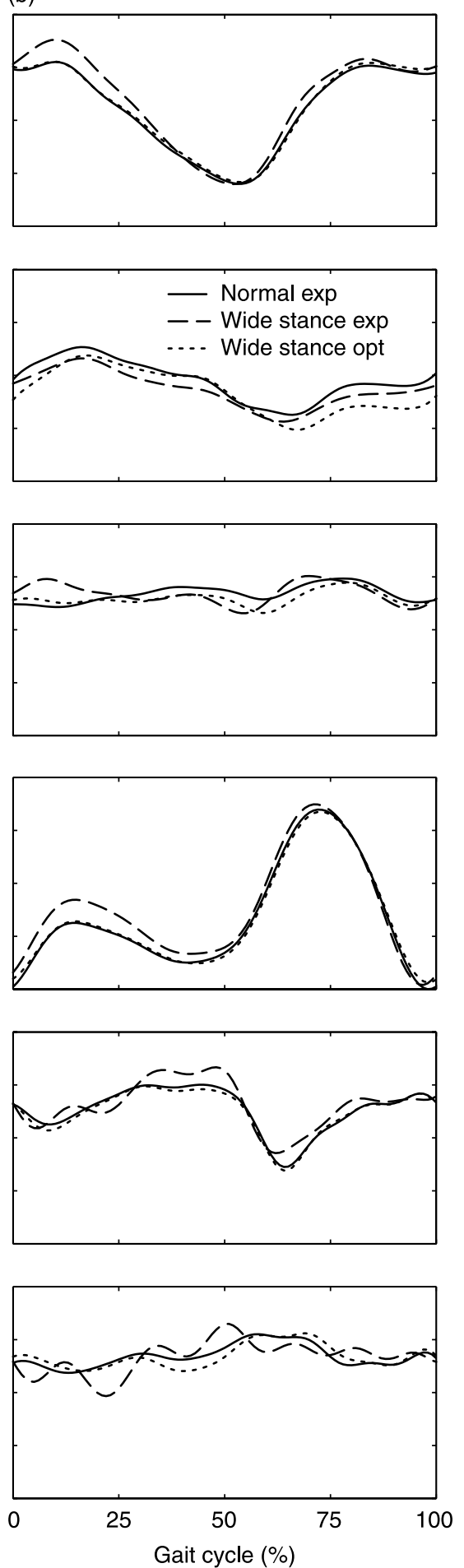

Figure 3. Evaluation of left leg joint angle curves predicted using the patient-specific optimization cost function. Column (a) Toe out gait prediction. Column (b) Wide stance gait prediction.

gait model can be used to predict how foot path changes will affect the patient's knee adduction torque. The optimization was formulated as an optimal tracking problem, where the cost function minimized the weighted sum of squares of changes away from the patient's nominal gait data. The cost function weights were calibrated to predict the patient's toe out gait adduction torque peaks and then tested by predicting his wide stance gait peaks. After calibration, a single set of cost function weights successfully predicted both adduction torque peaks to within $7 \%$ error for both toe out and wide stance gait. Many kinematic and kinetic changes (or lack thereof) were also predicted well by the optimizations (e.g. the lateral shift in the centre of pressure), though some 
(a)
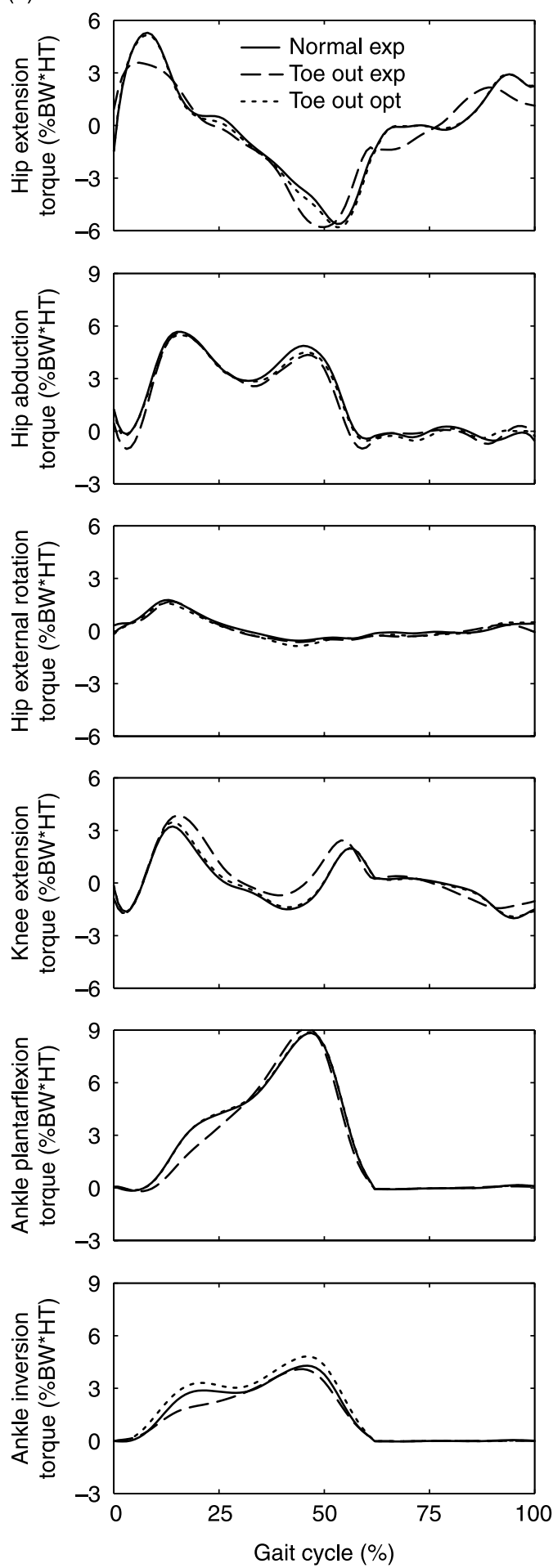

(b)
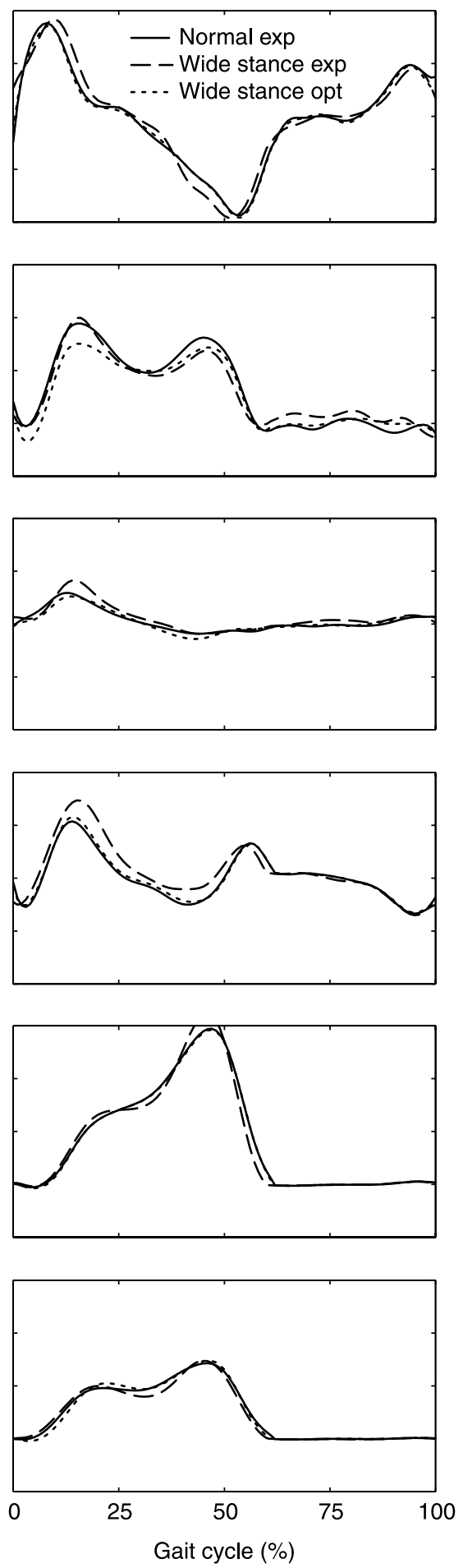

Figure 4. Evaluation of left leg joint torque curves predicted using the patient-specific optimization cost function. Column (a) Toe out gait prediction. Column (b) Wide stance gait prediction.

changes observed experimentally were not reproduced (e.g. the increase in knee extension torque). Thus, while the proposed cost function accurately predicted the quantity for which it was calibrated, it did not predict all potential quantities of interest accurately. Nonetheless, these initial results suggest that with future developments, a similar approach may make it possible to design rehabilitation or surgical treatments so as to maximize functional outcome for the individual patient.

The ability of a single set of cost function weights to predict the patient's adduction torque peaks for two different gait motions suggests that our initial hypothesis about neighbouring solutions was reasonable. One possible outcome was that no cost function weights could be found 
(a)
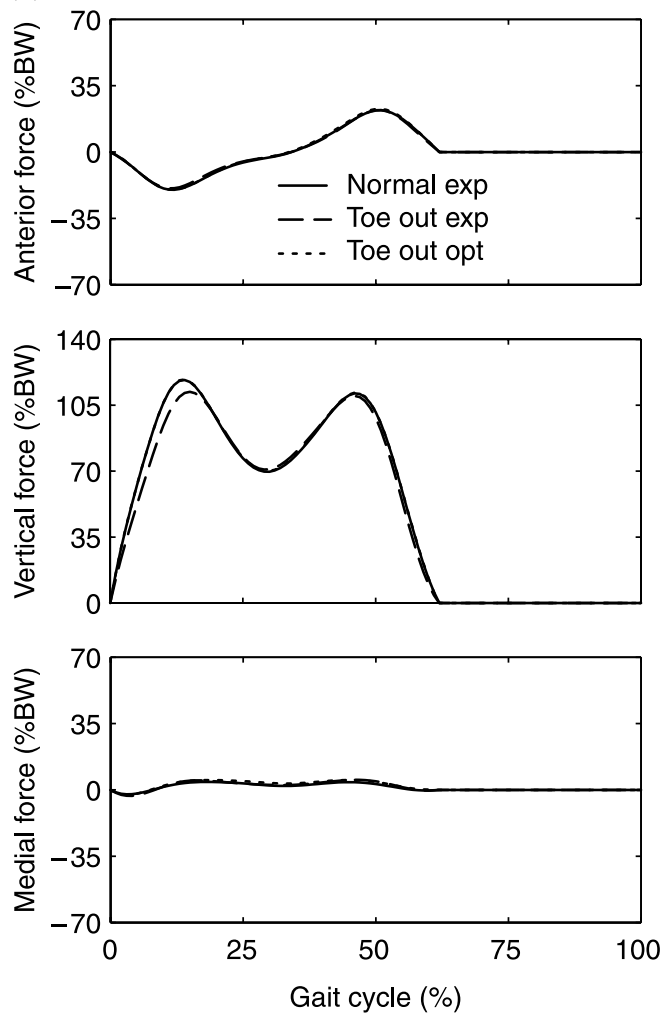

(b)
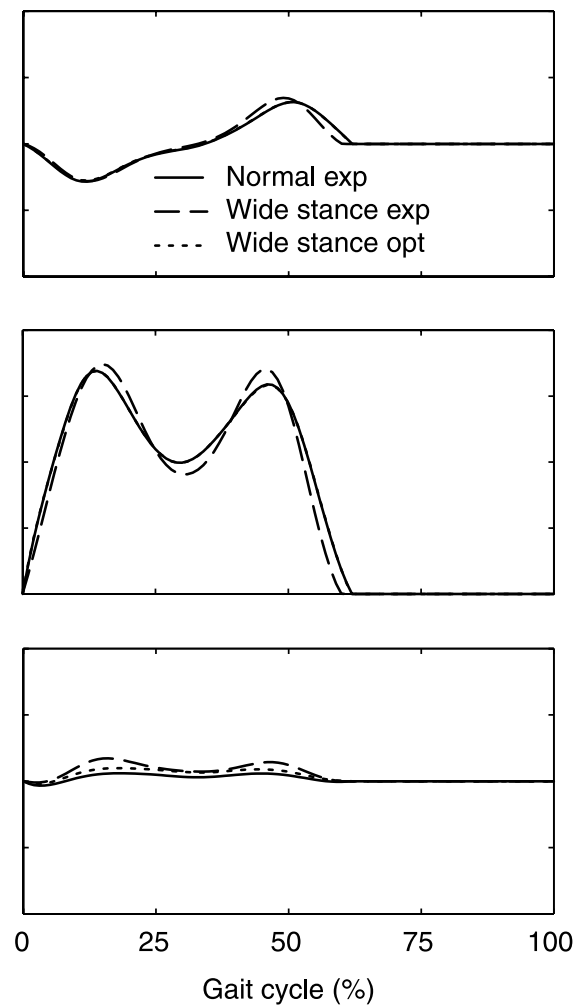

Figure 5. Evaluation of left leg ground reaction force curves predicted using the patient-specific optimization cost function. Column (a) Toe out gait prediction. Column (b) Wide stance gait prediction.

that successfully predicted both adduction torque peaks for toe out gait starting from the patient's normal gait motion. Another possibility was that the calibrated weights successfully predicted both adduction torque peaks for toe out gait but not wide stance gait. It was therefore encouraging that a single set of weights predicted both adduction torque peaks to within $7 \%$ error for two different foot paths, including one for which the weights were not calibrated.

Despite this result, the calibrated weights did not yield accurate predictions for all kinematic and kinetic quantities from the two gait motions with modified foot paths. This finding may indicate limitations in the model structure, errors in the model parameters, or (most likely)

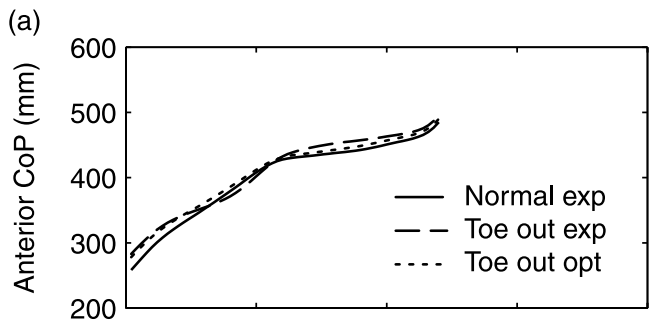

(b)
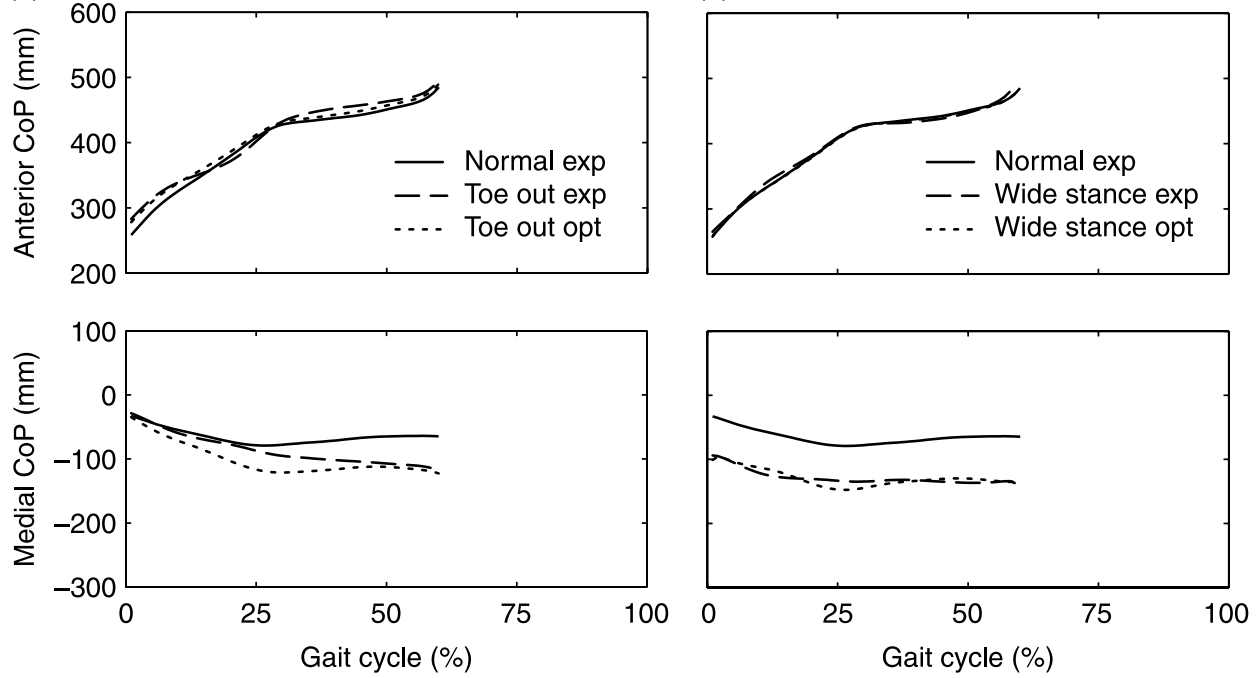

Figure 6. Evaluation of left foot centre of pressure curves predicted using the patient-specific optimization cost function. Column (a) Toe out gait prediction. Column (b) Wide stance gait prediction. 
the need for refinement in the cost function formulation. Two obvious refinements are worthy of future investigation. The first would be to put different weights on the different leg control torques, since different muscle groups possess different strength properties. The second would be to eliminate the need to use a normal data set for tracking purposes. While the assumption that new gait motions are neighboring solutions of the patient's normal gait motion appears to be reasonable, a more elegant solution would be to calibrate cost function weights to a single data set, as done in several recent studies (Liu et al. 2005, Bottasso et al. 2006). This latter approach could also address the limitation that the current cost function formulation does not have an obvious physical interpretation tied to a hypothesized neural control strategy.

One limitation of our two-level optimization approach is that the calibration process was extremely tedious. Each time the outer-level optimizer chose a new set of cost function weights, the inner-level optimizer had to perform a new optimization to determine the resulting errors in the predicted adduction torque peaks. Since each inner-level optimization required about $45 \mathrm{~min}$ of CPU time on a 1.7 GHz Pentium M laptop computer, iterating between the outer- and inner-level optimizers was an extremely time consuming task. A gradient-based optimizer was initially implemented at the outer-level, but numerical noise in the inner-level optimization results made accurate calculation of finite difference gradients difficult. Switching from forward to central differencing would have improved the accuracy of the gradient calculations but at the cost of double the computation time. The univariate search method used a bisection method that avoided the need for gradient information, though this approach was not highly efficient computationally.

Another limitation of our two-level optimization approach is that the results depend on which error terms are included in the cost function. This issue has been noted in previous studies that seek to determine patient-specific cost function weights for human movement optimization problems (Bottasso et al. 2006). In the present study, error terms were selected for inclusion based on experience gained from repeated optimizations performed with and without different terms. However, there is no simple way to determine whether all of the necessary terms were included, or whether omission of certain terms would not affect the predictions adversely. While one term could be omitted at a time, the current two-level optimization approach for calibrating the cost function weights is too inefficient to be repeated a large number of times. Future studies will investigate new methods for performing the calibration process in a more computationally efficient manner.

One possibility for making the calibration process more efficient would be to replace the inner-level optimization with a surrogate model. Surrogate models such as polynomial response surfaces are frequently used in structural optimization research to approximate the inputoutput relationships of a computationally expensive model
(Jansson et al. 2003). For the calibration process, the relationship between errors in predicted adduction torque peaks (i.e. the outputs) and values of cost function weights (i.e. the inputs) could be fitted using polynomial response surfaces or other surrogate modeling methods (e.g. Kriging, support vector regression; Queipo et al. 2005). Each time consuming inner-level optimization would then be replaced by extremely fast evaluation of a surrogate model (one for each adduction torque peak). To generate the surrogate model, one would use design of experiments (e.g. optimal Latin hypercube) to sample different combinations of weights. For each combination, an inner-level optimization would be performed to generate the corresponding outputs to be used in the surrogate model fitting process. Once the surrogate models were available, any type of outer-level optimizer (e.g. gradientbased, global) could be used to find the optimal set of weights in a matter of seconds or minutes. Surrogate models could also provide analytical derivative information, and new cost functions could be evaluated rapidly using additional outputs generated by the inner-level optimization sample points.

The patient-specific gait model used in the optimizations also possesses several limitations (e.g. no muscles, no ground contact model, rigid foot model), the pin joint knee assumption likely being the most important one. A single kinematic model was used to represent the patient under three separate gait conditions, with the optimized knee axis providing the best possible fit to the threedimensional knee motion from the normal gait data. It is possible that for this patient, who had slight anteriorcruciate ligament laxity, a single fixed knee axis was not an accurate representation for all three gait motions. This hypothesis would explain why the knee extension torque was predicted more poorly than were the other joint torques. However, reduction of the tracking weight on only the knee extension torque improved the prediction, making it is difficult to conclude whether the cost function or the model structure was the source of the problem. Regardless, the knee adduction torque was still well predicted, suggesting that the pin joint knee assumption had little influence on the predicted quantity of primary interest.

One final limitation of the study was that the optimizations were performed for only a single patient. Furthermore, only two gait patterns were predicted starting from the patient's normal gait data. While our initial results are encouraging, further evaluation with additional patients and gait patterns is needed to assess the limitations and general applicability of the approach.

In terms of future clinical application, an important issue is determining when a patient-specific cost function is, and is not, necessary. In some cases, the clinician may want to predict post-treatment function for given treatment parameters assuming the patient's neural control strategy does not change. For these situations, calibration of cost function weights is necessary to match what the patient will do naturally. Predicting how a specified change in foot 
path will affect a patient's knee adduction torque peaks is one example. In other cases, the clinician may want to optimize post-treatment function for unknown treatment parameters assuming the patient's neural control strategy can be changed. For these situations, calibration of cost function weights is not necessary, but the cost function must include additional terms related to clinical outcome (e.g. the knee adduction torque). Design of patient-specific gait modifications to minimize both knee adduction torque peaks is a recent successful example (Fregly et al. 2007). When changes in neural control are permitted, some type of novel real-time feedback system may be necessary to help patients adapt their gait to the predicted optimal gait patterns.

In conclusion, this study has demonstrated that a patient-specific cost function combined with a patientspecific gait model can be used to predict clinically significant gait characteristics under conditions for which experimental data are not available. Though the approach was evaluated with a single patient, demonstration of feasibility is a critical first step toward application to a larger number of patients. While a different cost function formulation will likely improve the predictive capabilities of the approach, the current results suggest that patientspecific musculoskeletal models may soon permit rehabilitation and surgical treatments to be designed and optimized on an individual patient basis, at least for a limited set of clinically important conditions.

\section{Acknowledgements}

The author gratefully acknowledges the support of the Whitaker Foundation to perform this study.

\section{References}

F.C. Anderson and M.G. Pandy, "Dynamic optimization of human walking", J. Biomech. Eng., 123, pp. 381-390, 2001.

A.S. Arnold, S. Salinas, D.J. Asakawa and S.L. Delp, "Accuracy of muscle moment arms estimated form MRI-based musculoskeletal models of the lower extremity", Comput. Aided Surg., 5, pp. 108-119, 2000 .

A.J. Baliunas, D.E. Hurwitz, A.B. Ryals, A. Karrar, J.P. Case, J.A. Block and T.P. Andriacchi, "Increased knee joint loads during walking are present in subjects with knee osteoarthritis", Osteoarthritis Cartilage, 10, pp. 573-579, 2002

A.J. Bogert, G.D. van den Smith and B.M. Nigg, "In vivo determination of the anatomical axes of the ankle joint complex: an optimization approach", J. Biomech., 27, pp. 1477-1488, 1994.

C.L. Bottasso, B.I. Prilutsky, A. Croce, E. Imberti and S. Sartirana, "A numerical procedure for inferring from experimental data the optimization cost functions using a multibody model of the neuromusculoskeletal system", Multibody Syst. Dyn., 16, pp. 123-154, 2006.

T.S. Buchanan, D.G. Lloyd, K. Manal and T.F. Besier, "Neuromusculoskeletal modeling: estimation of muscle forces and joint moments and movements from measurements of neural command", J. Appl. Biomech., 20, pp. 367-395, 2004.

P. de Leva, "Adjustments to Zatsiorsky-Seluyanov's segment inertia parameters", J. Biomech., 29, pp. 1223-1230, 1996.

B.J. Fregly, H. Rahman and S.A. Banks, "Theoretical accuracy of modelbased shape matching for measuring natural knee kinematics with single-plane fluoroscopy", J. Biomech. Eng., 127, pp. 692-699, 2005.

B.J. Fregly, J.A. Reinbolt, K.L. Rooney, K.H. Mitchell and T.L. Chmielewski, "Design of patient-specific gait modifications for knee osteoarthritis rehabilitation", IEEE Trans. Biomed. Eng., 54, pp. 1687-1695, 2007.

B.A. Garner and M.G. Pandy, "Estimation of musculotendon properties in the human upper limb", Ann. Biomed. Eng., 31, pp. 207-220, 2003

T. Jansson, L. Nilsson and M. Redhe, "Using surrogate models and response surfaces in structural optimization-with application to crashworthiness design and sheet metal forming", Struct. Multidiscip. Optim., 25, pp. 129-140, 2003.

C.K. Liu, A. Hertzmann and Z. Popovic, "Learning physics-based motion style with inverse optimization", ACM Trans. Graph., 24, pp. 1071-1081, 2005.

D.G. Lloyd and T.F. Besier, "An EMG-driven musculoskeletal model to estimate muscle forces and knee joint moments in vivo", J. Biomech., 36, pp. 765-776, 2003

C. Mazza and A. Cappozzo, "An optimization algorithm for human joint angle time-history", Ann. Biomed. Eng., 32, pp. 764-772, 2004.

T. Miyazaki, M. Wada, H. Kawahara, M. Sato, H. Baba and S. Shimada, "Dynamic load at baseline can predict radiographic disease progression in medial compartment knee osteoarthritis", Ann. Rheum. Dis., 61, pp. 617-622, 2002.

W.M. Murray, T.S. Buchanan and S.L. Delp, "Scaling of peak moment arms of elbowmuscles with upper extremity bone dimensions", J. Biomech., 35, pp. 19-26, 2002.

M.L. Nagurka and V. Yen, "Fourier-based optimal control of nonlinear dynamic systems", J. Dyn. Syst. Meas. Control, 112, pp. 17-26, 1990.

N.V. Queipo, R.T. Haftka, W. Shyy, T. Goel and R. Vaidyanathan, "Surrogate-based analysis and optimization", Prog. Aerosp. Sci., 41, pp. $1-28,2005$

G. Rao, D. Amarantini, E. Berton and D. Favier, "Influence of body segments' parameters estimation models on inverse dynamics solutions during gait”, J. Biomech., 39, pp. 1531-1536, 2006.

J.A. Reinbolt, J.F. Schutte, B.J. Fregly, R.T. Haftka, A.D. George and K.H. Mitchell, "Determination of patient-specific multi-joint kinematic models through two-level optimization", J. Biomech., 38, pp. 621-626, 2005

H.J. Sommer, III and N.R. Miller, "A technique for kinematic modeling of anatomical joints", J. Biomech. Eng., 102, pp. 311-317, 1980.

M. Suzuki, K. Matsunami, Y. Yamazaki and N. Nizuno, "Application of the minimum jerk model to formation of the trajectory of the centre of mass during multijoint limb movements", Folia Primatol., 66 , pp. 240-252, 1996.

D.G. Thelen, F.C. Anderson and S.L. Delp, "Generating dynamic simulations of movement using computed muscle control", J. Biomech., 36, pp. 321-328, 2003.

C.L. Vaughan, J.G. Andrews and J.G. Hay, "Selection of body segment parameters by optimization methods", J. Biomech. Eng., 104, pp. 38-44, 1982

Y. Wada, Y. Kaneko, E. Nakano, R. Osu and M. Kawato, "Quantitative examinations for multi joint arm trajectory planning-using a robust calculation algorithm of the minimum commanded torque", Neural Networks, 14, pp. 381-393, 2001.

F.E. Zajac, "Muscle and tendon: properties, models, scaling, and application to biomechanics and motor control", CRC Crit. Rev. Biomed. Eng., 17, pp. 359-411, 1989.

D. Zhao, S.A. Banks, K.H. Mitchell, D.D. D'Lima, C.W. Colwell and B.J. Fregly, "Correlation between the knee adduction torque and medial contact force for a variety of gait patterns", J. Orthop. Res., 25, pp. 789-797, 2007. 